\title{
Research and Analysis Based on Electricity Spot Market Construction in Northern China
}

\author{
Sen Wang ${ }^{1 *}$, Shuai Zhao ${ }^{2}$, Liansheng Zhou ${ }^{1}$, Zhiyong Gan ${ }^{1}$, Changzhi Zhang ${ }^{1}$, Yue Zhao ${ }^{1}$, Dengxin Ai ${ }^{1}$, Yigang Zhou ${ }^{1}$ \\ ${ }^{1}$ Electric Power Research Institute of State Grid Tianjin Electric Power Company, Zip Code:300384, Xiqing District, Tianjin, China \\ ${ }^{2}$ State Grid Tianjin Electric Power Company, Zip Code:300010, Hebei District, Tianjin, China
}

\begin{abstract}
Successive development of China's power spot market has subjected the operation and dispatching mode of the existing power system to an increasingly greater influence imposed by the electricity market mechanism. In China's northern provinces requiring winter heating, it is a huge challenge to the trading mechanism of power spot market to simultaneously guarantee the safe operation of power system and the heating mode of heating unit. To address this issue, this paper analyses the operation conditions and problems of power spot market during winter heating in northern China. Based on the analysis of the power spot market in a northern city, This paper analyzes some crucial problems in the construction of spot market and sums up policy advice for the further construction of spot market adapted to direct electricity purchase by large consumers in northern China.
\end{abstract}

\section{Research Background}

As an important part of the electricity market, spot market plays a fundamental supporting role for the opening, competition and orderly operation of the electricity market and is crucial to coordinating market transaction and system security. Therefore, there is a pressing need to analyze power market mechanism based on the latest practice and dissect the system structure and how it is constructed. In-depth discussion about the intrinsic logic and major problems of spot market construction is also needed so as to provide useful decision-making basis for the further reform of electricity market in northern China.

\section{The Forming Mechanism of Spot Electricity Price}

\subsection{The Nodal Electricity Price Mechanism}

Nodal electricity price, also known as locational marginal price (LMP), is defined as the variation of the total power purchase cost when one unit of electricity load is increased on a certain node. Under the circumstance where line transmission constraints are not taken into consideration or the line transmission capacity is large enough to avoid congestion, the system is capable of calling any unit in the network to meet the demand of the node load. In the order of bidding, the last successful unit that can meet the slightly increased load is the marginal unit. The nodal electricity price of the entire system is equivalent to the system marginal clearing price, namely the marginal unit price. The occurrence of line congestion makes it impossible to call the cheaper marginal units. Consequently, these nodes can only acquire power from the more expensive units through other non-blocking lines, rendering price on each node in the system different. Influenced by line congestion, the electricity price of certain nodes is even higher than the quote of the highest bidder or lower than that of the lowest bidder.

\subsection{The Clearing Mechanism of Spot Market}

The clearing mechanism of power spot market is illustrated in Figure 1. Spot market clearing means that market operators, with the aid of technical support system and on the basis of the boundary conditions of grid operation and information declared by market members, employ security constrained unit commitment (SCUC) and security constrained economic dispatch (SCED) to execute optimal calculations, and close the transaction after clearing. The determination of unit combination-SCUC model and the power generation plan and the nodal electricity price-SCED model are as shown respectively in Table 1 and table 2 .

*Corresponding author's e-mail: wangsen429@163.com 


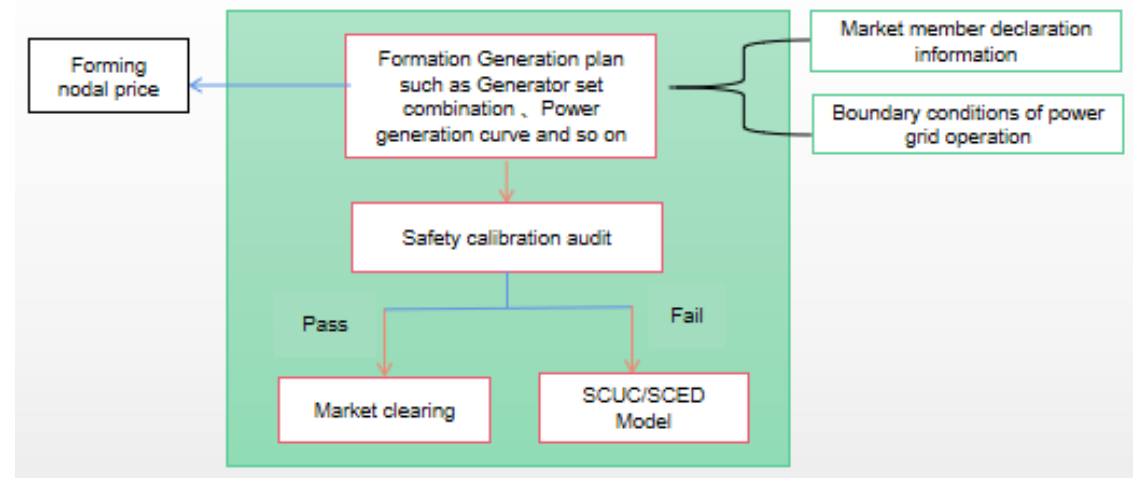

Figure 1. The clearing mechanism of spot market

Table 1. The determination of unit combination - SCUC model

\begin{tabular}{|l|l|}
\hline \multicolumn{1}{|c|}{ objective of optimization } & \multicolumn{1}{c|}{ constraints } \\
\hline \multirow{3}{*}{$\begin{array}{l}\text { the lowest power generation } \\
\text { cost in the entire grid }\end{array}$} & $\begin{array}{l}\text { unit constraints: constraints on the upper and lower limits of output, } \\
\text { constraints on climbing rate, constraints on the minimum time of continuous } \\
\text { start-stop, constraints on the maximum start-stop times; } \\
\text { system constraints: load balancing constraints, system backup constraints; } \\
\text { network constraints: constraints on line power flow, constraints on } \\
\text { cross-section transmission limit. }\end{array}$ \\
\hline
\end{tabular}

Table 2. The power generation plan and the nodal electricity price - SCED model

\begin{tabular}{|l|l|}
\hline \multicolumn{1}{|c|}{ objective of optimization } & \multicolumn{1}{|c|}{ constraints } \\
\hline \multirow{3}{*}{$\begin{array}{l}\text { the lowest power generation } \\
\text { cost in the entire grid }\end{array}$} & $\begin{array}{l}\text { unit constraints: constraints on the upper and lower limits of output, constraints } \\
\text { on climbing rate; } \\
\text { system constraints: load balancing constraints, system backup constraints; } \\
\text { network constraints: constraints on line power flow, constraints on } \\
\text { cross-section transmission limit. }\end{array}$ \\
\hline
\end{tabular}

\subsection{The Forming Process of Spot Price}

The price forming mechanism in spot market is expounded in the following examples which refer to three power generation enterprises in northern China. In this condition the load is presumed to be $710 \mathrm{MW}$.
Each power generation enterprise has to declare volume-price information. The volume price trend appears as a monotonous non-decreasing curve of multiple segments that reveals volume-price information. Unit:yuan/MWh. The basic behaviour and quotation information of each power generation enterprise are as shown in Table 3 and Table 4 respectively:

\subsubsection{Market Declaration}

Table 3. The basic behaviour of each power generation enterprise

\begin{tabular}{|c|c|c|}
\hline Power generation enterprises & Installed capacity (MW) & Minimum technical output (MW) \\
\hline A & 250 & 125 \\
\hline B & 150 & 75 \\
\hline C & 500 & 250 \\
\hline
\end{tabular}

Table 4. The quotation information of each power generation enterprise

\begin{tabular}{|c|c|c|}
\hline Power generation enterprises & Quoted capacity segment $(\mathrm{MW})$ & Price $($ yuan/MWh $)$ \\
\hline \multirow{4}{*}{$\mathrm{A}$} & {$[125,180]$} & 250 \\
\cline { 2 - 3 } & $(180,210]$ & 425 \\
\cline { 2 - 3 } & $(210,250]$ & 625 \\
\hline \multirow{3}{*}{$\mathrm{B}$} & {$[75,100]$} & 350 \\
\cline { 2 - 3 } & $(100,125]$ & 525 \\
\cline { 2 - 3 } & $(125,150]$ & 650 \\
\hline \multirow{3}{*}{$\mathrm{C}$} & {$[250,300]$} & 100 \\
\cline { 2 - 3 } & $(300,400]$ & 600 \\
\cline { 2 - 3 } & $(400,500]$ & 280 \\
\hline
\end{tabular}




\subsubsection{The Price Forming Mechanism}

The capacity of each segment of all power generation enterprises is sorted in the order of price from the lowest price to the highest without considering safety constraints. The purpose is to minimize the sum of the output operation cost and the start cost produced by generator set. When the accumulative output meets the load demand, the last winning bidder, namely the successful unit quoting the highest, ranks as the system marginal unit which possesses the pricing power. The price quoted by the marginal unit is the system marginal price. The forming mechanism of clearing price without constraints is as shown in Figure 2. Without safety constraints, the system marginal price of an unconstrained system is 525 yuan/MWh. Power plant B is the system marginal unit which possesses the pricing power. The quoted capacity segments of power plant $\mathrm{C}(400,500], \mathrm{A}(210,250]$ and $\mathrm{B}(125,150]$ will not be called due to overpricing.

In contrast, if safety constraints are considered, the capacity of each segment of all power generation enterprises is still sorted in the order of price from the lowest price to the highest. Then in this condition, such constraints on the branch power flow, the upper and lower limits of unit processing, and the regulation rate of unit active power must be considered during market clearing. Under the circumstances network constraints might prevent the units from being called despite the lower quotations of certain units. The clearing results with safety constraints considered are as shown in Figure 3. The system marginal price here is 600 yuan/MWh, and power plant $\mathrm{C}$ acts as the system marginal unit. The quoted capacity segments of power plant $\mathrm{A}(180,210]$ and $(210,250]$ can not be called due to the overload of safety constraints. The quoted capacity segments of power plant $\mathrm{B}(125,150]$ will not be called due to overpricing. It can be observed that the existence of safety constraints would make the unit unable to be called even if its price appears lower than the quoted capacity segment of power plant A(180, 210].

It can also be perceived that price rather than tap position serves as the sort criterion. When safety constraints are taken into consideration, a lower quotation is not the sufficient condition for itself to be called. If situation arises where safety constraints can not be met, units offering lower price might suffer from clearing failure, while units offering higher price will be called, which will elevate the system marginal price.

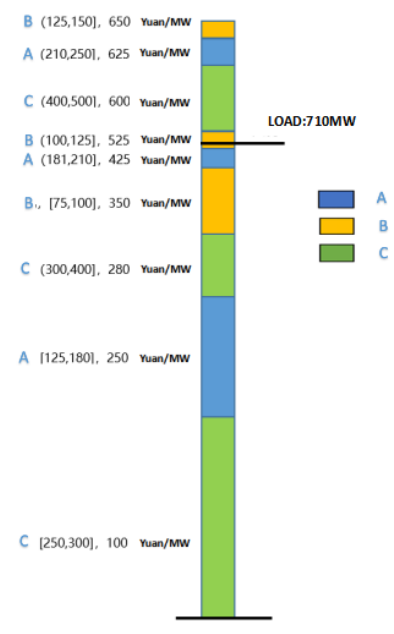

Figure 2. The forming mechanism of clearing price without constraints

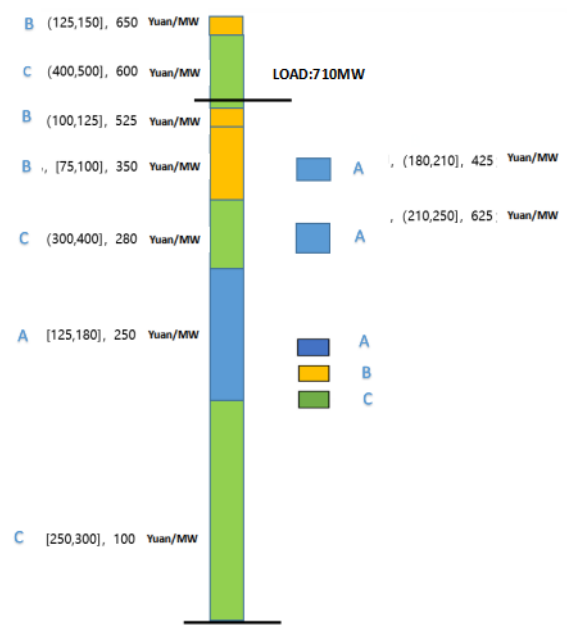

Figure 3. The forming mechanism of clearing price with constraints considered

\subsubsection{The System Marginal Price Curve of Daily Clearing}

The above has explained the forming mechanism of clearing price at a single load level. On that basis, the following example referring to 12 daily load levels introduces the clearing mechanism of time-sharing price. Load level is shown in Figure 5.

Table 5. Daily load level

\begin{tabular}{|c|c|c|c|c|c|c|c|c|c|c|c|c|}
\hline No. & 1 & 2 & 3 & 4 & 5 & 6 & 7 & 8 & 9 & 10 & 11 & 12 \\
\hline $\begin{array}{l}\text { load level } \\
\text { (MW) }\end{array}$ & 710 & 650 & 675 & 735 & 810 & 655 & 755 & 785 & 660 & 685 & 725 & 740 \\
\hline
\end{tabular}

The clearing results without safety constraints are as shown in Figure 4. The black line in the figure represents the daily load curve. It is indicated that the system marginal price is time-sharing price. A higher demand of system load is accompanied by a higher system marginal price after market clearing, which proves that the time-sharing system marginal price mechanism is capable of reflecting market supply and demand, and mirroring the value of power generation resources. The time-sharing system marginal price is shown in Table 4, while the time-sharing correlation between market demand and market price is illustrated in Figure 5. 
The clearing process of the system marginal price and the trend of clearing price fluctuation are similar regardless of the consideration or exclusion of safety constraints. Nevertheless, the market clearing price with safety constraints considered might differ from that without constraints due to safety constraints.

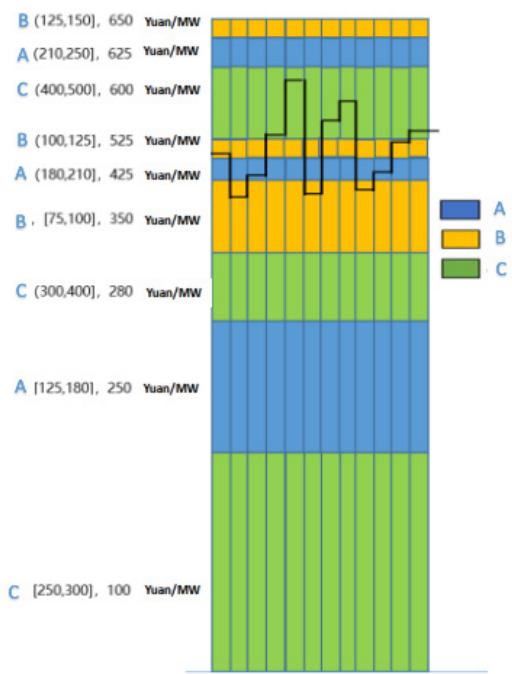

Figure 4. Clearing results without constraints

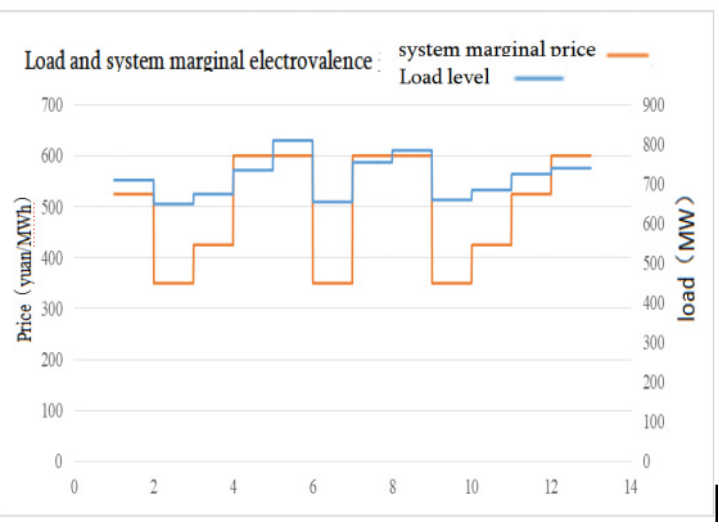

Figure 5. Diagram of load and system marginal electricity price

Table 6. The time-sharing system marginal price

\begin{tabular}{|c|c|c|c|}
\hline Number & $\begin{array}{c}\text { Load level } \\
\text { (MW) }\end{array}$ & Pricing unit & $\begin{array}{c}\text { system marginal } \\
\text { price(yuan/MWh) }\end{array}$ \\
\hline 1 & 710 & $\begin{array}{c}\text { Power plant } \\
\text { B }\end{array}$ & 525 \\
\hline 3 & 675 & $\begin{array}{c}\text { Power plant } \\
\text { A }\end{array}$ & 425 \\
\hline 4 & 735 & $\begin{array}{c}\text { Power plant } \\
\text { C }\end{array}$ & 600 \\
\hline
\end{tabular}

\section{Analysis and advice on spot market construction}

\subsection{Selection of the spot market clearing method}

In the spot market, especially in clearing calculation, market players are often required to declare the bilateral transactions they've signed to market organizers who make a unified optimization match between power generation resources and electricity demand of the whole network. However, not all the electricity has been re-cleared in spot market. Market players can declare the bilateral transactions they wish to be physically executed in advance. The declared electricity will remain unchanged as the external input condition in the central clearing calculation of the spot market.

\subsection{Selecting transaction clearing-based physical model}

What physical model should be adopted in spot market transaction clearing mainly depends on the physical operation condition of the power system. A system with good resource endowment and network condition means that it has sufficient resource supply, adequate ancillary service adjustment capability, light network congestion and weak fluctuation of new energy. Under such circumstance, the physical model of the spot market can be simple. Only some crucial constraints need to be considered or the clearing can be done without any constraint. For the system with adequate resources and adjustment capability, the optimal dispatching of safe operation prioritizes the spot market. On the contrary, if the system has insufficient resources or serious network congestion, the realistic physical model of the system should be taken into account in the spot market. Besides, optimal clearing should be done to the power and ancillary service resources and lowering various security risks that may be encountered in real-time operation is also needed. The physical model used in clearing calculation is often consistent with the spot market. Because the main function of the model is to balance dispatching and manage congestion of the system, the most accurate physical model should be used in clearing calculation. Real operating conditions and network constraints of various equipment should be taken into full consideration. Market players should be required to report the actual physical parameters, for example, power generators must declare technical parameters like the climbing rate, maximum and minimum output and continuous start-stop time constraints.

\subsection{Determination of transaction scale in spot market}

The transaction scale of different market links is formed naturally in the process of market cultivation. The scale is closely related to the functional orientation, rule design, transaction cost and convenience of each link. So, it is not reasonable to artificially determine the transaction scale. But given that China is still at the primary stage of the development of electricity market, the transaction scale of spot market can be gradually liberalized through certain policy intervention.

\subsection{Construction of Spot Market}

Spot market is the market carrier of the medium and long-term direct power purchase transaction delivery, reflecting the transaction price. The power generation and consumption plan will be an important basis for the 
real-time dispatching of power grid. The deviation between real-time dispatching curve and day-ahead power generation and consumption curve will be made an ex-post settlement according to rules. Spot market has little impact on the dispatching and safety of market in northern China, thus it can be given priority. At the same time, since spot market is the last "gate closure" connecting market transaction and physical operation of the system, under the premise of ensuring security, market mechanism can be introduced and be developed after the spot market becomes mature.

\subsection{Physical Model and Price Mechanism of the Spot Market Clearing}

As for the physical model and price mechanism adopted for clearing transactions in the spot market, the actual physical model of power grid and the physical and technical parameters of the units and equipment should be taken into consideration so as to ensure the fitting between the transaction plan and the actual dispatching. Security check of the day-ahead market should be strengthened and the optimal allocation of power resources should be guided by locational marginal price. At the same time, on the basis of carrying out day-ahead electricity trading, transactions should be organized according to the backup and frequency regulation resources and gradually couple with electricity trading and finally realize integrated transaction clearing of main and ancillary electricity resources, thus boosting the optimal utilization of electricity resources.

To sum up, spot market is an important part of the electricity market. According to the situation of the power industry in northern China, we should introduce the market-oriented reform idea of power supply side competition by considering various aspects like the determination of the spot market trading scale, the system construction, selection of the transaction physical model and design of the price mechanism so as to realize the optimal utilization of power resources.

\section{Acknowledgments}

This work was financially supported by "Science and Technology Project of State Grid Tianjin Electric Power Company (KJ20-1-46 Reaserch on the Fluctuation of the Price of Electricity Spot Market under the Uncertainties of Source-grid-load and Its Countermeasures)".

\section{References}

1. Evolution of EROIs of electricity until 2050: Estimation and implications on prices[J]. Adrien Fabre. Ecological Economics.

2. The impact of major fuel retailers on regional New South Wales petrol prices[J]. Edward Oczkowski, Alfred Wong, Kishor Sharma. Economic Analysis and Policy.

3. Commodity boom fuels product prices[J]. China Rare Earth Information. 2017(09)
4. Optimization and Design of Construction Route for Electricity Market in China [J]. Ge Rui, Chen Longxiang, Wang Yiyu, Liu Dunnan. Automation of Electric Power Systems. 2017(24)

5. Review of Nordic Electricity Market and Its Suggestions for China[J]. Bao Minglei, Ding Yi, Shao Changzheng, Song Yonghua. Proceedings of the CSEE. 2017(17) 